\title{
Effects of image intensifier halo on perceived layout
}

\author{
James E. Zacher ${ }^{\mathrm{a}}$, Tracey Brandwood ${ }^{\mathrm{a}}$, Paul Thomas ${ }^{\mathrm{b}}$, Margarita Vinnikov ${ }^{\mathrm{a}}$, Gancun Xu ${ }^{\mathrm{a}}$, Sion \\ Jennings $^{\mathrm{c}}$, Todd Macuda ${ }^{\mathrm{c}}$, Stephen A. Palmisano ${ }^{\mathrm{d}}$, Greg Craig ${ }^{\mathrm{c}}$, Laurie Wilcox ${ }^{\mathrm{a}}$, Robert S. Allison ${ }^{\mathrm{a}^{*}}$ \\ ${ }^{\mathrm{a}}$ Centre for Vision Research, York University, 4700 Keele St, Toronto, ON, Canada M3J 1P3 \\ ${ }^{\mathrm{b}}$ Topaz Technology Inc, Toronto, Canada \\ ${ }^{c}$ National Research Council of Canada, Flight Research Laboratory, Ottawa, Canada \\ ${ }^{\mathrm{d}}$ University of Wollongong, School of Psychology, Wollongong, NSW, Australia
}

\begin{abstract}
Night vision devices (NVDs) or night-vision goggles (NVGs) based on image intensifiers improve nighttime visibility and extend night operations for military and increasingly civil aviation. However, NVG imagery is not equivalent to daytime vision and impaired depth and motion perception has been noted. One potential cause of impaired perceptions of space and environmental layout is NVG halo, where bright light sources appear to be surrounded by a disc-like halo. In this study we measured the characteristics of NVG halo psychophysically and objectively and then evaluated the influence of halo on perceived environmental layout in a simulation experiment. Halos are generated in the device and are not directly related to the spatial layout of the scene. We found that, when visible, halo image (i.e. angular) size was only weakly dependent on both source intensity and distance although halo intensity did vary with effective source intensity. The size of halo images surrounding lights sources are independent of the source distance and thus do not obey the normal laws of perspective. In simulation experiments we investigated the effect of NVG halo on judgements of observer attitude with respect to the ground during simulated flight. We discuss the results in terms of NVG design and of the ability of human operators to compensate for perceptual distortions.
\end{abstract}

Keywords: Night vision devices, Human factors, Depth Perception, Slant, Halo, Perspective, Layout

\section{INTRODUCTION}

Night vision devices (NVD or Night Vision Goggles, NVGs) are critical to nighttime operations for military aviators and ground forces. The devices allow forces to 'own the night' by intensifying ambient illumination, providing visibility under reduced light conditions. However image intensifiers do not provide daytime equivalent vision and the devices suffer from a number of limitations or artifacts. For example, the image is monochromatic, contaminated by image noise at low light levels, the unusual spectral sensitivity can result in contrast inversions and field of view is limited in most devices. These limitations and artefacts presumably underlie the reported deficits in perception of space, depth and motion. One artefact of NVG viewing is the halo that surrounds images of bright light sources in the display.

Halo in the context on NVGs refers to the phenomenon that a bright light source viewed through NVGs appears to be surrounded by a corona or halo that is much larger than predicted by the point spread function of the device. The phenomenon is superficially similar to the physiological halo reported in normal and diseased eyes and the coronas that can be seen when viewing light sources through the atmosphere. However, NVG halos are generated in the image intensifier tubes. Examination of these halos is important for both NVG design and to understand limitations on their use in operational settings ${ }^{1}$. Being device artifacts, these halos have characteristics that are significantly different from the associated environmental features in the image. These distinctions are perceptually important and predict specific distortions of perceived environmental layout and movement. We provide quantitative description of the halos formed by light sources as a function of intensity and distance and describe briefly a method and preliminary data from psychophysical experiments designed to analyse halo-induced errors in slope estimation.

*allison@cse.yorku.ca

Head- and Helmet-Mounted Displays XII: Design and Applications edited by Randall W. Brown, Colin E. Reese, Peter L. Marasco, Thomas H. Harding Proc. of SPIE Vol. 6557, 65570U, (2007) · 0277-786X/07/\$18 - doi: 10.1117/12.719892

Proc. of SPIE Vol. 6557 65570U-1 


\subsection{Halo Phenomenology}

There are considerable anecdotal reports and descriptions of the phenomenology and effects of NVG halo but published data in the open literature is sparse. The effective brightness of a light source depends on intensity, direction and spectral content. If a bright light, such as a NVG incompatible vehicle light, is viewed in an NVG then the user typically reports seeing the image of the light source surrounded by disc-like halo. The brightness of the disc appears to depend on the intensity of the light source and can appear transparent for relatively weak lights allowing for visibility of scenery beyond the halo. With current technology, halo is a ubiquitous feature of both the built environment (Figure 1) and natural scenes (e.g. in starfields). While halo like effects can be observed surrounding bright light sources - for example with corneal edema - the presence of halos around numerous features in a visual scene is both an unusual and unnatural stimulus.

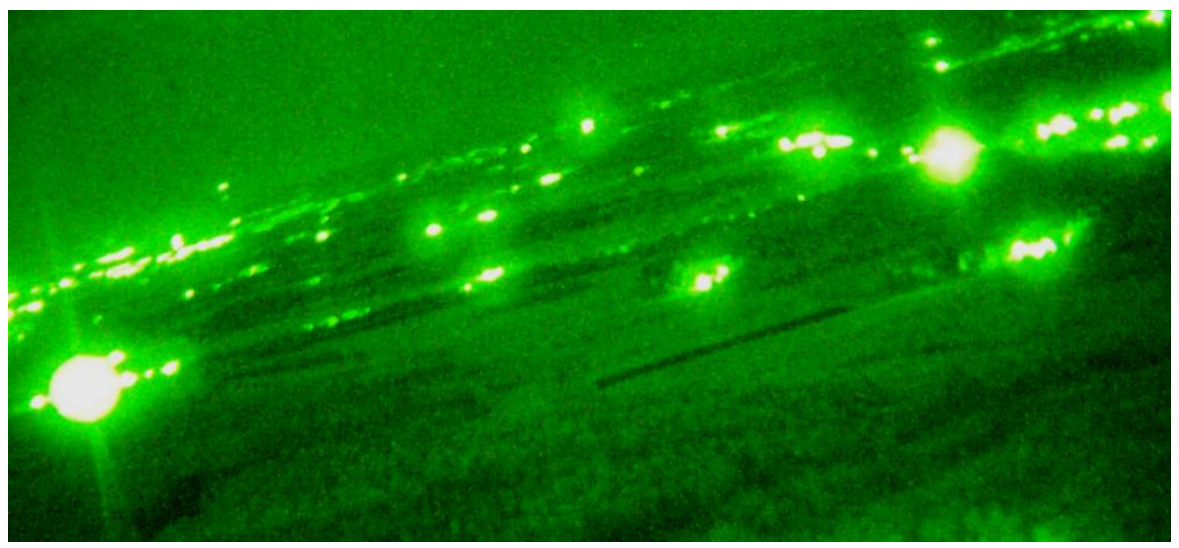

Fig. 1. NVG halos surround a variety of bright point light sources in a scene. Where light source density is high, such as in the upper right hand corner, halos may merge. Note that the apparent whitening and widening of the central spot in very bright halos is due to camera nonlinearity and saturation and is not apparent when viewing by eye. Typically when viewing a bright point source with NVGs the light source appears small and distinct but surrounded by a larger 'disc'.

Informally, we have noted that halo angular size in the image is largely independent of light source intensity and distance. That is, once a light is bright enough to produce a halo then the halo appears. Increase in source intensity increases the brightness of the 'disc' and diminishes its transparency. However, the halos of a very bright and moderately bright source appear to have the same angular diameter. With very bright sources the primary halo appears to be surrounded by a weaker secondary halo. NVG images may have other artifacts created by bright light sources and care must be taken not to confuse these with halo. Halos of nearby or extended sources can merge and form extended halos surrounding the extended configuration.

\subsection{Halo Metrics}

Metrics such as halo intensity, transparency, symmetry, and profile shape and their dependence on source intensity, distance, shape and spectral characteristics are important but relatively unexplored in the open literature. Our group has two papers on the subject of halo image size that need to be reconciled.

In Thomas et $\mathrm{al}^{2}$, halos were created and measured under controlled laboratory conditions with the eyepiece replaced by a CMOS camera. Halo image size increased slowly with increasing intensity over a range of target intensities spanning 7 orders of magnitude. Craig et $\mathrm{al}^{3}$ had subjects compare the apparent linear size of the halo generated by a fixed intensity LED light source located at distances of 4.5 to $22.5 \mathrm{~m}$ within a set of concentric rings. These rings served as a gauge figure, surrounding the target. The basic psychophysical result was that apparent halo angular size was independent of viewing distance. The actual judged variable of apparent linear halo size was of course scaled by distance and thus halos appeared to be smaller objects in the scene when produced by a nearer source. Image measurements from digital photographs taken of the subject's view confirmed the subjective responses in that the halo image size on the NVG output screen (which is proportional to it angular size) did not vary with distance.

There are a number of important differences between the Thomas et al. and Craig et al. studies that make them difficult to compare. The former used a single near target distance and varied intensity over a wide dynamic range while the intensity variation in the latter study was modest and was accomplished by varying the target distance. The precision of 
the gauge figure and CorelDraw based image measurements used in the Craig study was relatively coarse and could not be used to detect the small changes in halo size with intensity expected from the Thomas study. The ambient illumination required to see the gauge figure could also have masked changes in halo size. In neither study was there any distinction between single and double halo phenomena. Furthermore, in the Thomas study we cannot be sure that a perceptual halo was present since psychophysical measurements on equivalent conditions were not available in this optical bench setup. Finally it is entirely possible that observers may use a different perceptual strategy than the thresholding scheme used in the objective measurements in Thomas et al (e.g. observers may be sensitive to the shape of the halo profile). Precise subjective (psychophysical) and objective measurements under equivalent conditions are required to resolve this issue. In comparing the two studies one must assume that the principle effect of changing the distance of a point source is a change in the effective intensity. Measurements of halo size while independently varying distance and intensity could help justify this assumption.

\section{VARIATION IN HALO SIZE WITH SOURCE DISTANCE AND INTENSITY}

Evaluation of the perceptual effects of halo depends on an understanding of their image characteristics. The effective halo generating potential of a point source is presumed to be a function of ambient illumination, source intensity, spectral content and distance. We designed a study to compare perceptual and objective measures of NVG halo size directly using identical laboratory conditions.

\subsection{Methods}

A custom built light source and optical bench were designed and built to present variable intensity stimuli at a range of distances. The subject's head was supported in a head and chin rest and placed in front of the NVG eye piece (see Figure 2). The NVG was a standard ANVIS-9 with GenIII image intensifier tubes.

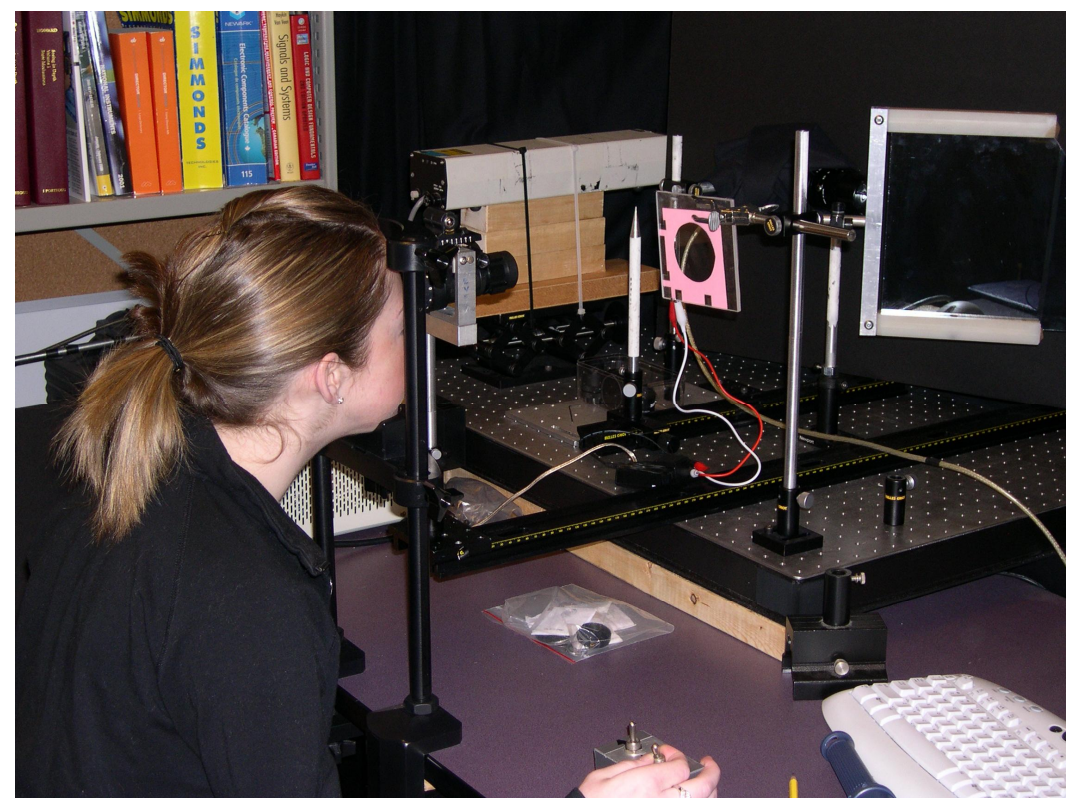

Fig. 2. View of subject seated in experimental apparatus, which extended for $8 \mathrm{~m}$ to the subject's right along a visual lane that was directed to the subject's view via a beamsplitter (top left). Matte cloth and material used to eliminate stray light have been removed for visibility in this photo.

The target light source was an LED mounted in a custom housing and driven by a custom driver board under computer control. A small $0.5 \mathrm{~mm}$ aperture was mounted at the output of the LED source to ensure the target could be regarded as a point source. An alignment laser was used to centre the light source, one tube of the NVGs and the appropriate baffles and apertures on the optical axis of the optical bench. The NVG objectives were focused at the target distance. A camera could be introduced into this light path to document or verify the alignment of the source and other elements. A stylised view of the setup is shown in Figure 3. 


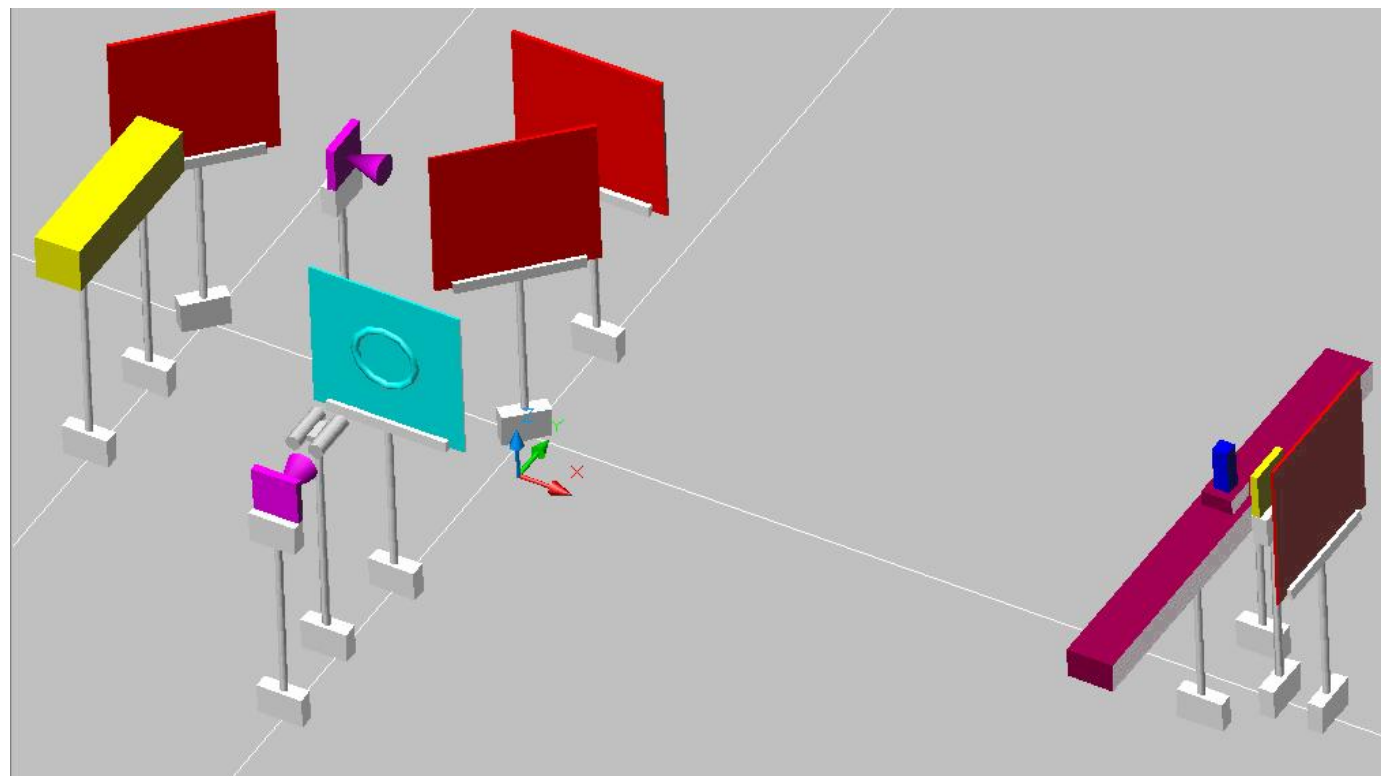

Fig. 3. - Schematic view of the test setup with target at $2 \mathrm{~m}$. Eye point/measurement camera (pink) is located behind the NVGs (gray tubes) looking through aperture (cyan) and mirror (magenta) at target at the far right (yellow plate). At the left of the rendering is shown a second camera (pink) and alignment laser (large yellow block) that were used for alignment and monitoring. The illuminated line (blue rectangle) on a long baseline linear stage (magenta) was used to measure the halo size.

Custom Labview code was written to control the experiment and collect the data. Driving the LED with a pulse-width modulated digital signal permitted a wide range of light source intensity. The PWM frequency $(1000 \mathrm{~Hz})$ was sufficient such that no flicker was observed. The code also randomised trials and sequenced and recorded the data from experimental trials.

In separate blocks of trials the target light was presented at one of three distances from the nodal point of the NVG objective $(2.0,4.0$ and $8.0 \mathrm{~m})$ at approximately the centre of the NVG field of view. At each distance the target was presented at one of five intensity levels scaled for viewing distance. The target was either presented in darkness (the room was blackened and extraneous light baffled and suppressed with matte black cloth, paint or paper) or in the presence of an illuminated surround. The illuminated surround filled the periphery of the NVG field of view but did not illuminate the target. A gap of 10-15 degrees was placed between the edge of the surround and the target. The purpose of the surround was to study the effect of the NVG AGC on halo size. Thus there were 30 different conditions ( 3 distances X 5 intensity levels X 2 background illumination conditions). These conditions were repeated five times per subject resulting in 150 measurements per subject within a counterbalanced design to control for any order effects.

For each condition we made three measurements in separate trials. Two measurements were subjective and intended to measure apparent halo angular size (image size as opposed to linear size in the world) and the third was an objective measure using a digital camera. All measures were cross calibrated to each other and to standard targets at known distances to get commensurate data in terms of visual angle at the NVG.

The first subjective measurement was direct and used a fine-grained reticule mounted in the NVG eyepiece as a gauge. The $18 \mathrm{~mm}$ reticule had horizontal and vertical scales extending $5 \mathrm{~mm}$ from the centre that were marked in steps of $5 \mu \mathrm{m}$ per minor division with major divisions marked and enumerated every 10 steps. The cross hair formed by the intersection of the axis was centred on the target and the subject was required to estimate the radius of the halo in terms of the number of divisions covered.

The second subjective measurement used an approach similar to a stage micrometer. A long-travel, motorized linear translation stage was mounted with its direction of travel perpendicular to the viewing direction just in front of the light source (fixed together on a rigid plate that could be moved between the viewing distances). Mounted to the stage was a long, vertically oriented illuminated line (formed by LEDs) that could be translated horizontally in front of the target. Care was taken that the line did not halo (intensity has been increased in figure 7 and the line used was much dimmer). 
At the beginning of each trial the line was aligned visually with the halo-producing target. Then the subject moved the stage outward until the inside edge of the illuminated line of the stage micrometer was aligned with the perceived edge of the halo (a precise Vernier alignment task). The stage was moved with a stepper motor and an encoder was used to measure the stage position with a resolution of 2048 counts per $\mathrm{cm}$ of translation. The measurements were repeated starting well outside the halo region to account for hysteresis effects. The halo diameter was estimated by comparing the distance between the indicated left and right edges of the halo.

For the objective measurements, a Nikon Coolpix 5400 digital camera was placed in the position of the subject's eye and used to image the NVG output through the ocular. The camera is based on a 2,592x1,944 pixel colour CCD sensor. The camera was set in manual focus within the shutter priority control mode and a short focal length was chosen from the camera range of 5.8 to $24 \mathrm{~mm}$ ( $35 \mathrm{~mm}$ equivalents $28-116 \mathrm{~mm}$ ). The camera was shrouded to prevent light contamination.

Five LED intensities were chosen for each distance to provide overlap in equivalent intensity ranges between distances and to produce a wide variety of halos (none, single, and double). The intensity of each stimulus was measured by a photometer through the NVG.

\subsection{Objective Measures}

Examples of images captured by the digital imager are shown in Figure 4. Typically as light intensity increased the image changed from showing a small spot to having an obvious halo to showing signs of double halos.
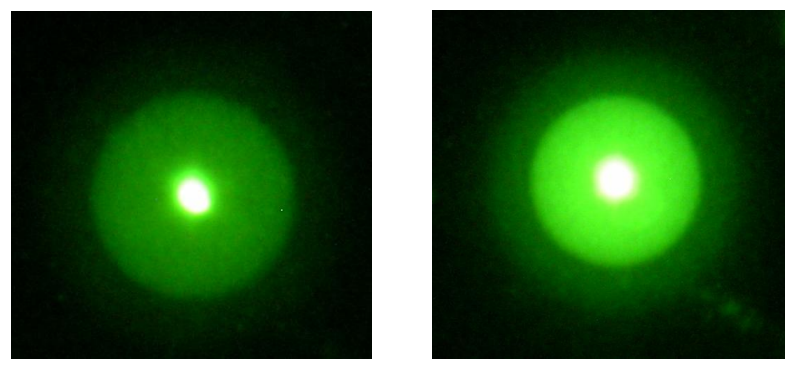

Fig. 4. Typical halo imagery for two intensities. In the left hand image the bright central spot is centered on the point source target and a 'disc' halo appears to surround the spot. A secondary halo is apparent but much more pronounced in the right hand image. Dynamic range limitations of the camera are apparent in the saturation of the bright central spot.

To estimate the halo widths the centre of mass of the image of the light source was calculated. Then cross-sections at one degree intervals were made through the centre of the spot and averaged to reduce noise (see Figure 5). Estimates of the half-width of the principal halo were based on the distance between maxima in change in slope in these cross-sections and plotted in Figure 6 as a function of intensity for both the background and no background conditions.

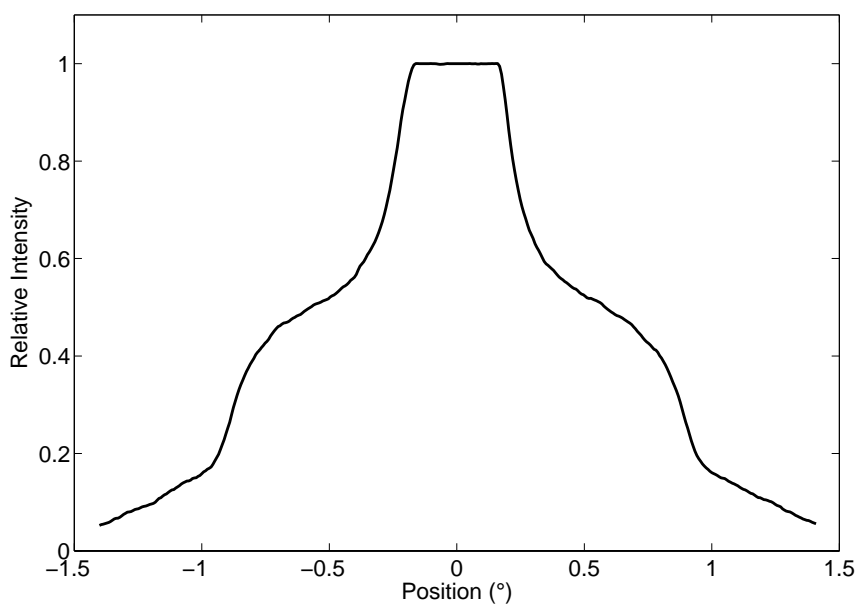

Fig. 5. Average cross-section through an NVG halo. 


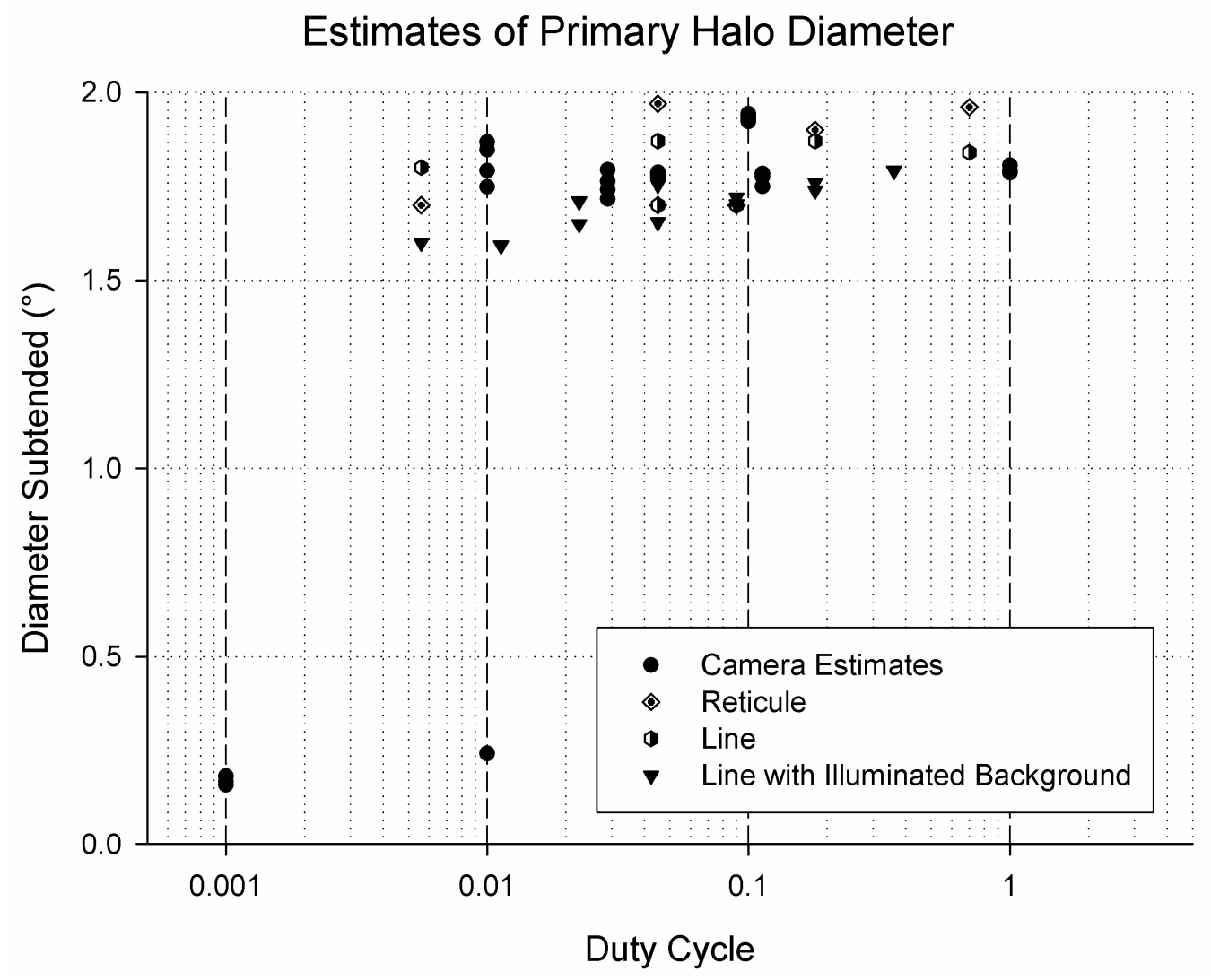

Fig. 6. Estimated halo width as a function of intensity for both the background and no background conditions.

In can be seen that halo size is approximately $1.7^{\circ}$ when halo is present and that there is no consistent variation in intensity despite a four-fold variation in distance and a thousand-fold change in intensity. Small estimates at the lowest intensity correspond to situations where a primary halo could not be detected in the image. Moving a real object from $8 \mathrm{~m}$ to $2 \mathrm{~m}$ would have resulted in a four-fold increase in image size whereas halo size does not vary with distance.

\subsection{Subjective Measures}

Representative views of the stimulus for the line alignment task are shown in Figure 7. Subjects found the task easy with estimated precision of 3 minutes of arc which compares well with reported values of Vernier acuity in the literature ${ }^{4}$ given the precision of the mechanical movement. The reticule task was easy when intensity was high since the target back-lit the scale. In dim light subjects found the task more demanding as the scale was difficult to see.

Both the reticule and halo measures were consistent with the objective data and indicated a perceived halo of roughly 1.7 degrees of visual angle (Figure 6). Responses at $2 \mathrm{~m}$ were slightly smaller than at 4 and $8 \mathrm{~m}$ but this reduction was less than 5\%. More data would be required to determine if this effect was reliable and if it was perceptual in origin. Background lighting drove the automatic gain control of NVG effectively. Subjects were less likely to perceive single or double halos with the background light than without at any given intensity. However, the background light did not appear to have a significant effect on the halo size. Halo estimates were variable with the AGC engaged and the subjects reported that the halo edge was less distinct and the judgements more difficult. 

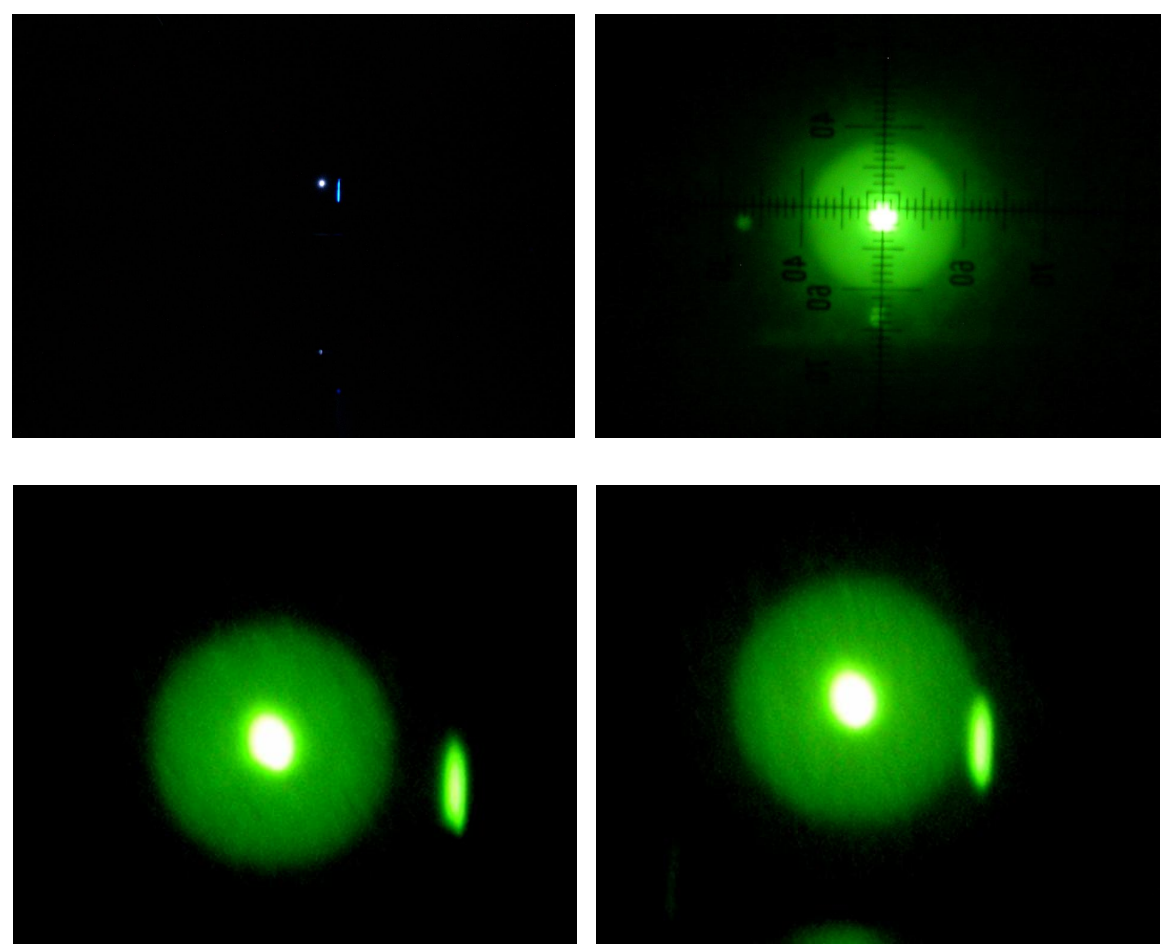

Fig. 7. Vernier alignment or stage-micrometer based halo measurement. Top left shows naked eye view. The bottom panels show magnified views of the NVG output showing round halo around the point source centre with the measurement line in various positions during the process of aligning with a single halo. Note that brightness of the line was increased relative to experimental conditions for photographic purposes. The top right panel shows the reticule with a source generating a double halo. Note how difficult it is to see the reticule scale when not back illuminated by the source. Normally the reticule was not present when making Vernier judgements. The photographs were taken by hand and are subject to camera linearity effects and thus should only be taken as an approximation of the subject's view.

\subsection{Discussion}

Two subjective measures and one objective measure of halo size were made using targets at a range of distances and intensities. These measures showed substantial agreement and equivalent conclusions can be drawn. NVG halo size is effectively invariant of light source intensity and distance when the halo is perceived. If a point source is bright enough to generate a halo then the size (but not intensity or transparency) of that halo is effectively constant with respect to distance and intensity - at least until secondary halos are seen. Any change in apparent size is small compared to the more salient effects of halo disappearance or double halo appearance as the source intensity is decreased or increased respectively (we assume that the principal effect of distance is on effective intensity). Halo intensity profile falls with eccentricity from the centre of the spot but is remarkably flat over the 'disc' potion of the halo. This effect needs to be modeled precisely but provides justification for our simple disc model of halo in section 3.

We found congruent results between two subjective measures and one objective measure. The objective camera measures provide data for modeling efforts and allow halo characterization with known and repeatable algorithms and parameters. Typical cameras have significant limitations particularly in terms of dynamic range that introduce imaging artifacts such as color shifts in peaks due to saturation and other non-linear effects ${ }^{2}$. The subjective measures are precise and use the eye, which is the intended imaging device. The line alignment/stage microscope technique is a 'field-based' measurement that matches halo size with a physical interval in the scene. The precision of the technique increases with distance as the linear size corresponding to a given angular size increases with distance although accomplishing the movement becomes more challenging. The reticule measurement is an image-based measure that measures perceived angular size directly. The reticule measure is similar to the technique reportedly used in industry where test markings are etched or deposited on the tube output itself. The latter has the advantage of excellent focus but also requires sufficient ambient light to illuminate the scale. Informally we have found the reticule technique to be an excellent indicator of halo size when looking at a natural scene but less precise and more difficult than the line alignment task in the laboratory. 
How do scene characteristics affect the halo images that are generated (the physical halo images - perceptual effects are considered in the next section)? Presumably, the principal parameter controlling halo generation is effective source intensity. This allows us to generalize to larger distances and to natural scenes. Typically the device is focused at a far distance (i.e. optical infinity) and depth of field is not an issue beyond a few meters. If the distance is large enough that the target is effectively a point source within the device's depth of field, then distance cannot be a determinant of halo size per se beyond its effect on effective source intensity. Similarly, the effective intensity of a source depends on its spectral characteristics and the wavelength selectivity of the NVG photocathode. Finally, the effective intensity is also a function of the 'gain' of the NVG, which is determined by scene illumination. As discussed above, the source intensity affects the likelihood that a primary and secondary halo will be perceived and the vividness of the halo but has little effect on halo image size when halo is present.

The NVG model developed by Thomas et $\mathrm{al}^{2,5}$ could readily be extended to incorporate the halo phenomena we have described with appropriate parameters such the threshold at which the halo appears, the spot profile, and the outer halo profiles and angular sizes. Our data were obtained using point light sources. Extended light sources appear to have a border that is haloed. Perhaps the phenomenon is linear and an extended light source can be considered a sum of point sources with the extended halo obtained from the superposition of the point source halos. Experiments with separated and overlapping halos from point sources could be informative.

It is not clear from our data whether the threshold for observing a halo is perceptual or a device characteristic. It may be that halo is always present but only perceived when its intensity exceeds visual threshold. Alternatively, the onset of the halo may represent some nonlinear process in the physical generation of the halo. Physics based modeling of the halo and additional experimentation may help elucidate this point. For example, if halo were a local nonlinear device phenomenon then we might expect that separated sub-threshold light sources would not combine to create a perceptible halo. Conversely, if sub-threshold halos were present, then in the overlap region we should expect an increased sensitivity to the summed visual stimuli.

\section{HALOS AS VISUAL STIMULI}

Based upon the halo phenomenology and data detailed above we can consider how halos may act as visual stimuli to influence the perception of objects and scene layout. To first order, a halo appears to be either present or not present and, if present, the size of the primary halo is invariant to variations in source intensity and distance. Important changes in the stimulus do occur with halo becoming brighter and less transparent as intensity increases and a secondary halo is often seen. The fact that primary halos do not change angular size with distance of the generating sources however lends them interesting perceptual properties. The size of images of real objects are of course a function of the distance of the object in accordance with the laws of perspective projection ${ }^{6}$. Halos are generated in the sensor and are in many respects similar to constant retinal images such as the afterimage seen when closing one's eyes after viewing a bright light. Such images are necessarily of a fixed retinal size. If one looks at a bright light and forms an afterimage on the retina and then gazes around an environment, then the afterimage will appear to change size depending on the distance of the surface on which the afterimage is projected. 'Emmert's law' describes how the apparent linear size of a retinal image depends on its perceived distance 7 . With halo (which are also of fixed image size), Emmert's law predicts that the apparent linear size of halos 'projected' onto the scene will increase with increased perceived distance and that a given halo will appear to shrink as one approaches the light source. Brightness is also a cue to distance and an isolated bright halo should appear nearer than a dim one even if further away. We have verified these predictions in the lab. It is important to note that complete size constancy is not to be expected and size constancy is reportedly poorer in NVG imagery than natural viewing ${ }^{8}$.

Besides affecting judgements of their depth and size, halos could have affects on the perception of the layout of the environment and surfaces within it. When considering three-dimensional surfaces one can parametrically describe the surface in terms of differential geometry related to surface orientation, curvature, rate of change of curvature, etc. For instance, local orientation in depth of a surface patch can be specified by the axis about which the surface patch is slanted and the angular degree of the rotation in depth about this axis - the tilt angle and the slant angle, respectively. The frame of reference can be fixed to the world (geographical slant) or with respect to person (i.e. oculocentric slant relative to the eye). Judgements of surface slant (and tilt) can be used by observers to estimate the orientation and layout of surfaces in the environment and can provide critical information to support helicopter low-level flight and landing tasks. When making judgements of slant humans rely on a number of visual cues including perspective, binocular disparity and motion parallax. 
One perspective-based cue that humans could use is known as texture gradient (Figure 8). If a homogeneously textured flat surface (such as a ground plane) is slanted in depth, then its retinal image will contain a gradient of texture element image size from near to far. This gradient will be manifest in the size of texture elements, their spacing, foreshortening and their density and will be present in NVG imagery. In the case of NVG halos, patterns of lights on the ground have an added texture corresponding to the halos generated by the NVG tubes. However, as the size of the halo is not related to source distance, texture element size (and shape) will be in conflict with perspective-based information in the scene.

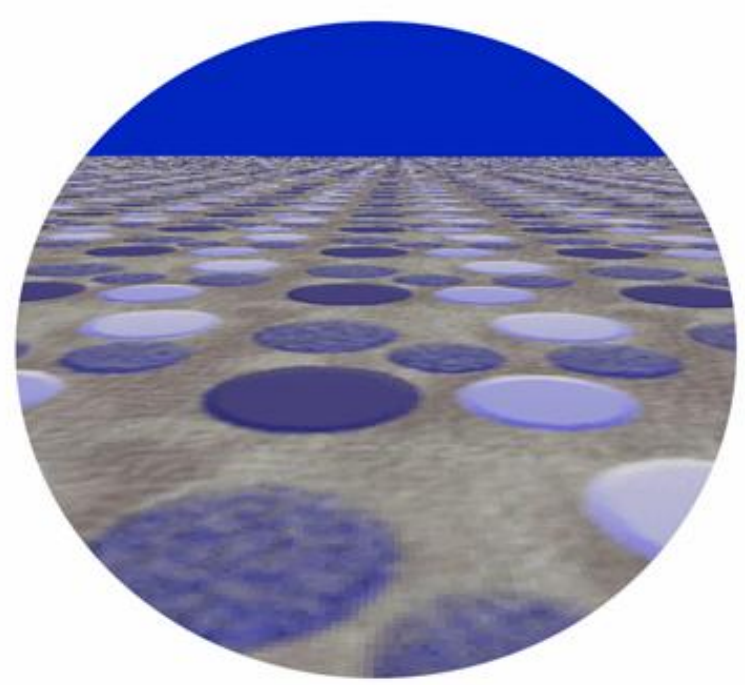

Fig. 8 Texture Gradients on Slanted Surfaces.

\section{HALO EFFECTS AND SLOPE JUDGEMENTS}

The effects of halo on judgments of slope were studied during simulated helicopter approach and landing. The halo size estimation data allows us to predict expected effects on texture gradients in the scene. We are modeling halo image size as invariant with distance, which is true to first-order in both our objective and perceptual halo measures. This specifies a texture gradient (more specifically a relative size cue) corresponding to a frontal surface and should produce cue conflict with other depth cues (including texture gradients from real textures), which, of course, scale in image size with depth according to the laws of perspective. The effects of this cue conflict on slant judgements are expected to be most pronounced when veridical cues are weak and minimal when strong cues to slant from texture, motion and binocular disparity exist. Conversely, when surface slant is correctly perceived, the halo should be interpreted as a feature in the environment.

Gibson $^{9}$ reported that subjects consistently underestimate the slant of surfaces defined by a texture gradient in the absence of other cues. He noted that this regression to the frontal plane was much stronger for irregular textures than for regular textures. One effect of texture irregularity is to add noise to estimates of texture gradient. Young et al. ${ }^{10}$ have provided evidence that under cue conflict, percepts shift to the more reliable cue when noise degrades information from the other. Changing perspective due to motion may be particularly compelling because stronger assumptions can be made than in the static case ${ }^{11}$. A static physically slanted surface could have texture that increases in size with distance. Homogeneity of texture element size is a fairly general assumption that is often invalidated in nature. An example would be a pebble beach where the pebbles are typically distributed by weight and thus size. On the other hand, when one moves relative to a static surface, changes in perspective (e.g. looming of texture elements) can specify the layout of the rigid scene independent of texture size, so the assumption of rigidity imposes a stronger constraint than texture homogeneity. Thus, in the dynamic case we may expect to see effects of superimposed halo on perceived surface slant 
rather than perceived texture size. In the following experiments we investigate the effects of superimposed halo on slant percepts when the surface is defined by regular or irregular patterns of lights and under static or dynamic conditions.

We have designed an experiment to test these predictions of the effects of NVGs on judgments of attitude with respect to the runway/landing pad. The goal is to estimate the effects of cue conflict on both the accuracy and the precision of ground slope estimates through NVGs. The experiments are being run in a custom-built stereoscope at our vision lab at York University and we present some preliminary data below.

\subsection{Methods}

Simulated helicopter approaches to a runway were presented in a large Wheatstone stereoscope. Mirrors mounted at +/45 degrees were located in front of the left/right eyes so that each eye viewed a large projection screen located to the side. Images were projected onto the screens via BARCO 808 projectors with a resolution of 1280x1024x100Hz. Each screen was driven by a separate graphics workstation in a Linux based graphics cluster. The video cards for the displays were genlocked and the simulations synchronized. The programs for efficiently displaying the imagery were custom designed based on Open/GL and Open/GL Shader language and the custom VE API developed at York University.

The runway approaches (or static views) are rendered using custom three-dimension computer graphics with imposed NVG effects from a physics-based model. Scenes were modeled in 3D Studio Max based on digital terrain maps (Figure 9). The modeled world contains a large flat plateau with a landing strip in the centre. The plateau was surrounded by simulated mountains that were unpredictable in location, height and distance on the plateau to prevent their being used as visual cues. The program was designed to allow for implementation of a flexible halo model. Various physical models of halo can be implemented but halo was initially modeled as a disk subtending a constant visual angle.

Subjects view simulated ground plane surfaces at various slants (via virtual camera pitch) and make judgments of surface orientation in depth. The surfaces are rendered as either stereoscopic or monocular images of simplified night scenes with halo or non-halo inducing light sources distributed on the ground plane. The intrinsic texture and perspective cues to depth in the scenes are varied by changing the regularity and configuration of the light sources.

The test scenes are either static, or depict simulated lateral motion or a simulated level flight approach. Subjects were instructed to estimate the slope of the ground (in pitch) with respect to the virtual camera. Following the stimulus a fullylit, full-cue daylight scene was displayed with random pitch angle. The subjects were given control of the pitch of the virtual camera and were asked to match the attitude of the virtual camera to their estimate. The match setting was recorded and the next trial began.

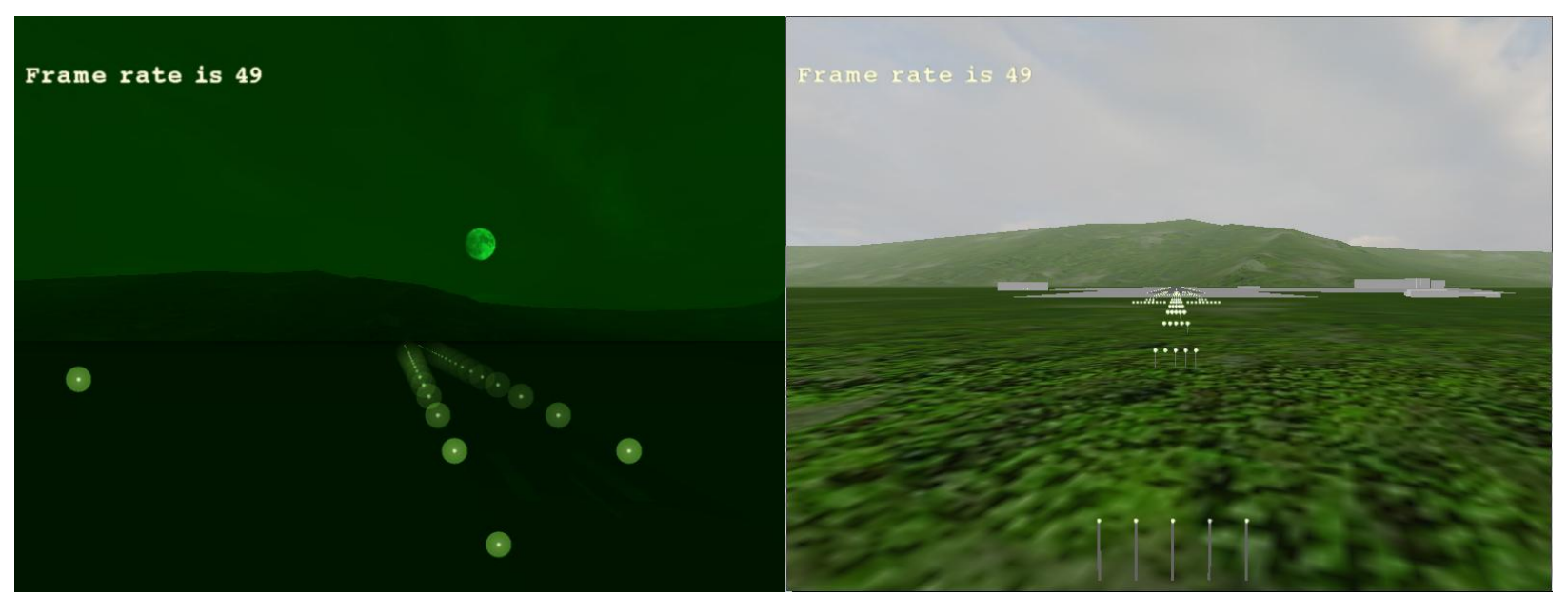

Fig. 9 Screen shots from simulated approaches. The light on the left of the left screen is isolated for illustrative purposes. The frame rate indication was turned off for the experiment and was one half the video refresh rate. 
The following manipulations were made in a factorial, repeated measures experiment: approach type (forward, lateral, static), aircraft pitch angle, light pattern (regular vs irregular), and lighting condition (day, night no halo, night with halo).

\subsection{Results}

Some preliminary data are shown in Figure 10. It can be seen that the subjects perceived increasing slant with increasing simulated slant in the daylight conditions (not shown) and in night time conditions with regular patterns of lights. Irregular patterns of lights resulted in a poor correlation between perceived and simulated slant. NVG halo effects are not very apparent in the data.

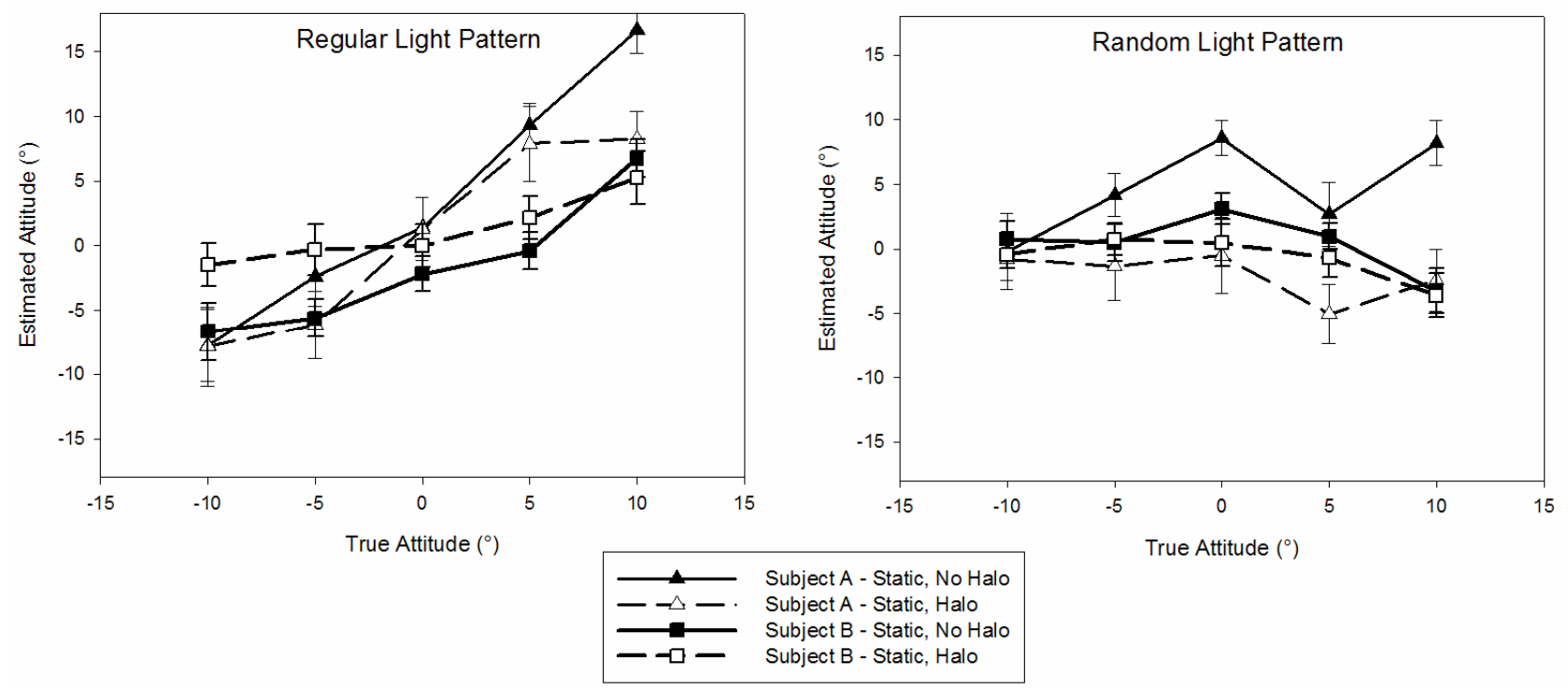

Fig. 10 Slant estimates as a function of halo and light pattern for two subjects.

When slant is seen in the regular light arrangement and halos are present, subjects report a strong impression of an increase in the perceived size of the halos with simulated distance although halos are constant size over the image. This is appropriate size constancy as found in Emmert's law. Anecdotally subjects report that they can 'see through' the halo to the slanted surface suggesting they can segregate the slant of the surface from the frontal slant specified by the halo.

\subsection{Discussion}

To date, we have found little evidence of an effect of halo on perceived slant. When slant cues in the scene were strong, subjects perceived slant that was near that found in the absence of halo as predicted. The regular pattern of lights provided a variety of perspective cues to depth including linear perspective, texture gradients, compression (and foreshortening) and the possibility of inferring an implicit horizon. When halos are present in a scene and associated with a slanted surface their size scales with apparent distance at least to an extent (size constancy). There is little conflict here as the strong slant cues dominate and the halo invariance is seen as a size gradient. Interestingly subjects viewing natural scenes sometimes report being able to see both a frontally oriented pattern of halos and to see through it to a slanted scene. Such dual percepts are sometimes seen in such cue conflict situations. van $\mathrm{Ee}^{12}$ has claimed that these dual percepts are alternating and bistable (like the famous Necker cube) although subjectively the impression is usually simultaneous rather than alternating.

Conversely the manipulation of using an irregular texture reduced slant percepts markedly both in halo and non-halo conditions. We had hypothesized a strong effect for halo scenes since the halo gradient would indicate a frontal surface. The manipulation may have been too strong, removing any reliable percept of surface slant change and thus there was no possibility of a halo effect (a floor effect).

We plan to continue these experiments using intermediate stimuli that may be more susceptible to a halo effect. We also plan to investigate the bimodal percept that can be seen in halo scenes. Finally we plan to look at cue conflict in scenes 
that are nearer frontal (i.e. a steep hill or cliff face) where the slants specified by the halo texture gradient (a frontal surface) and the true surface are more similar and thus more likely to be combined.

\section{CONCLUSIONS}

Objective and subjective measures of halo geometry indicate that halo size, when halo is present, is relatively invariant of target distance or intensity. These results predict systematic distortions of slant due to an imposed texture gradient. We investigated this hypothesis in a psychophysical experiment. Results to date are equivocal perhaps due to floor effects. Future experiments will address this issue, will incorporate increasingly more realistic physical halo models and will address active perception and control of glideslope during simulated landing in the presence of halo.

\section{ACKNOWLEDGEMENTS}

This work was performed for the NRC Flight Research Laboratory under PWGSC Contract \#561982 in support of the Advanced Deployable Day/Night Simulation Technology Demonstration Project headed by DRDC Toronto. Alex Tumanov and Jason Telner assisted in data collection for preliminary experiments related to this research.

\section{REFERENCES}

1 J. P. Estrera, T. Ostromek, W. Isbell, and A. Bacarella, "Modern Night Vision Goggles for Advanced Infantry Applications," Proc SPIE, 5079, 196-207, Orlando, FL, 2003.

2 P. J. Thomas, R. S. Allison, P. Carr, E. Shen, S. Jennings, T. Macuda, G. Craig, and R. Hornsey, "Physical modeling and characterization of the halo phenomenon in night vision goggles," Proc SPIE, 5800, 21-31, Orlando, FL, 2005.

${ }^{3}$ G. Craig, T. Macuda, P. Thomas, R. Allison, and S. Jennings, "Light source halos in Night Vision Goggles: Psychophysical assessments," Proc SPIE, 5800, 40-44, Orlando, FL, 2005.

4 S. P. McKee, L. Welch, D. G. Taylor, and S. F. Bowne, "Finding the common bond: stereoacuity and the other hyperacuities," Vision Research, 30, 879-91., 1990.

5 P. J. Thomas, R. S. Allison, S. Jennings, K. Yip, E. Savchenko, I. Tsang, T. Macuda, and R. Hornsey, "Validation of synthetic imagery for night vision devices," Proc. SPIE, 5442, 2535, Orlando, FL, 2004.

6 I. P. Howard, Rogers, B.J., Depth Perception, vol. 2. Pages, I. Porteous, Toronto, 2002.

7 E. Emmert, "Grossenverh,,ltnisse der Nachbilder," Klinische Monatsbl tter für Augenheilkunde, 19, 443-50., 1881.

8 A. M. Zalevski, J. W. Meehan, and P. K. Hughes, "Size estimation with night vision goggles," Aeronautical and Maritime Research Laboratory - Air Operations Division, Melbourne 2000.

9 J. J. Gibson, "The Perception of Visual Surfaces," American Journal of Psychology, 63, 367-384, 1950.

10 M. J. Young, M. S. Landy, and L. T. Maloney, "A perturbation analysis of depth perception from combinations of texture and motion cues," Vision Research, 33, 2685-96., 1993.

11 R. S. Allison and I. P. Howard, "Temporal dependencies in resolving monocular and binocular cue conflict in slant perception," Vision Research, 40, 1869-85, 2000.

12 R. van Ee, L. C. van Dam, and C. J. Erkelens, "Bi-stability in perceived slant when binocular disparity and monocular perspective specify different slants," J Vis, 2, 597-607, 2002. 